\title{
Motion of a Membrane Enzyme as Seen by SANS
}

\author{
W. Javed ${ }^{1,2,3}$, C. Ebel ${ }^{1}$, C. Orelle ${ }^{2}$, J.M. Jault $^{2}, \underline{\text { A. Martel }}^{3}$ \\ ${ }^{1}$ IBS; Univ. Grenoble Alpes, CNRS, CEA; Grenoble, 38000; France. \\ ${ }^{2}$ Molecular Microbiology \& Structural Biochemistry (MMSB) UMR 5086; CNRS/University of Lyon; Lyon, 69000; France, \\ ${ }^{3}$ Institut Max Von Laue Paul Langevin (ILL); Grenoble, 38000; \\ martela@ill.fr
}

Small Angle Neutron Scattering is a low-resolution technique enabling to probe the solution structure of individual biomacromolecules possibly in complex with its partners. In particular, concerning membrane proteins, the membrane-like environment can be made invisible in order to see only the protein. Here, we combined SANS with X-ray crystallography, cryoEM, $\mathrm{H} / \mathrm{D}$ exchange coupled with mass spectrometry and limited proteolysis to reveal the flexibility and ligand-induced conformational changes of the multidrug $\mathrm{ABC}$ transporter BmrA.

Limited proteolysis revealed an important flexibility of BmrA WT in most steps of its catalytic cycle. Cryo-EM provided highresolution of the closed conformation by analysis of an artificially monodisperse sample, and X-ray crystallography data enabled to build homology models of other conformations, which constituted the starting point of SANS analysis. H/D X-MS pinpointed the flexible part along the transporter sequence and SANS revealed the extent of this flexibility.

Together, these techniques enable us to describe the $\mathrm{ABC}$ transporter cycle in term of successive conformational equilibria, a much more realistic and accurate vision of this biological process [1].

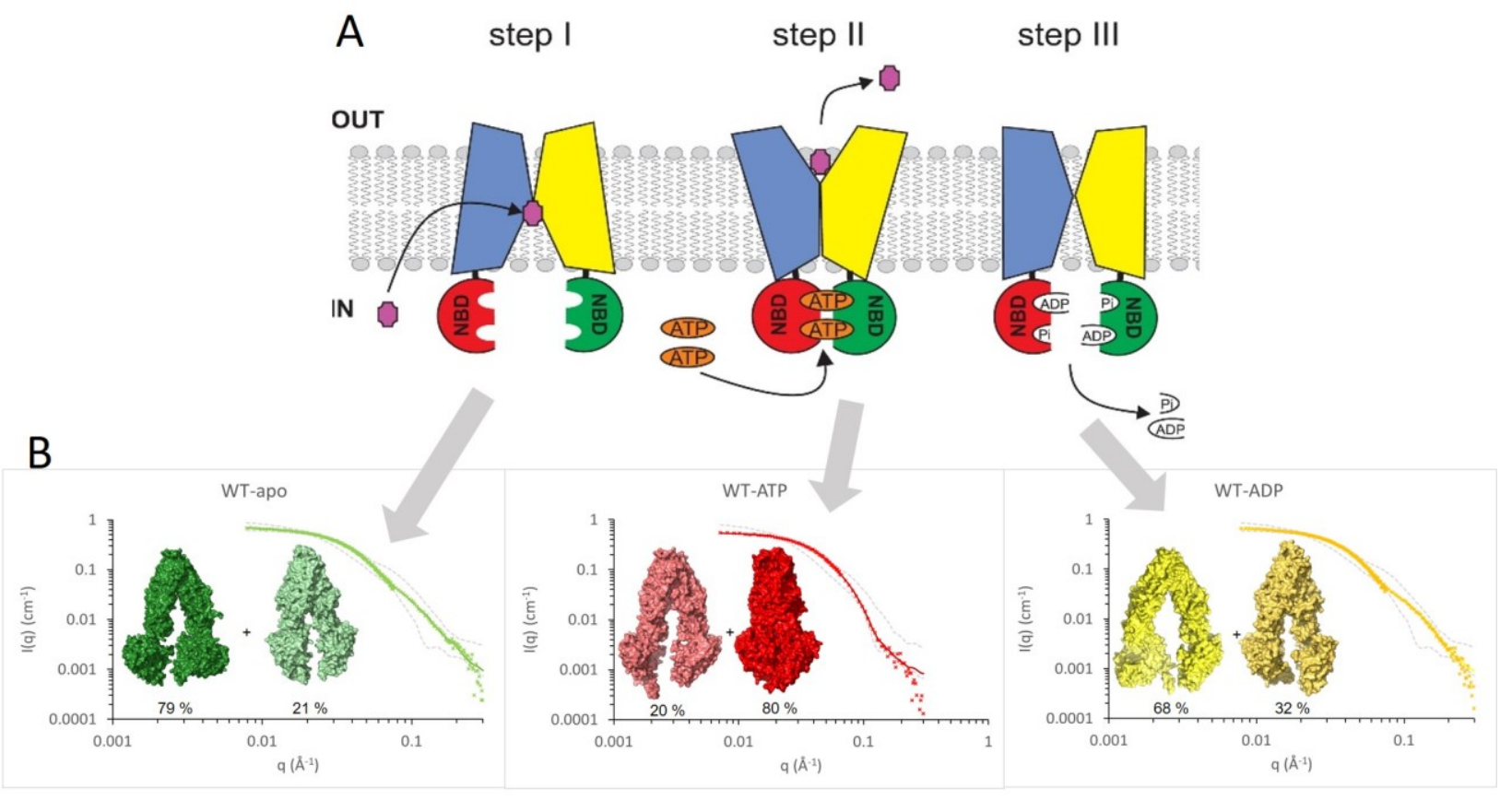

Figure 1. A: Main steps of the enzymatic cycle of ABC transporters (from [2]); B: Structural definition of these steps in solution by sequential conformational equilibria [1].

[1] Javed et al. in preparation (or submitted)

[2] Wannes Dermauw, Thomas Van Leeuwen, The ABC gene family in arthropods: Comparative genomics and role in insecticide transport and resistance, Insect Biochemistry and Molecular Biology, Volume 45, 2014

Keywords: Small Angle Neutron Scattering;Solution structure and dynamics; membrane protein; biological complexes

Acta Cryst. (2021), A77, C199 